[Vicino Oriente XXI (2017), pp. 1-3]

\title{
RICORDO DI GIOVANNI GARBINI \\ (1931-2017)
}

\section{Alessandro Catastini - Sapienza Università di Roma}

Il 2 gennaio 2017 è scomparso a Roma Giovanni Garbini. Nato a Fiastra (MC) l'8 ottobre 1931 e stabilitosi sin da giovanissimo a Roma, aveva qui condotto gli studi universitari a partire dagli inizi degli anni Cinquanta. Studente del corso in lettere classiche, aveva nutrito incertezze sul percorso definitivo da seguire, ponendoglisi la scelta tra l'archeologia classica, la letteratura indiana - una passione che risaliva all'adolescenza -, l'epigrafia classica e l'etruscologia: non di rado, in effetti, gli si sentivano ricordare con affetto Ranuccio Bianchi Bandinelli, Alfonsa Ferrari, Margherita Guarducci, Massimo Pallottino. L’incontro decisivo fu tuttavia con Sabatino Moscati, del quale decise di frequentare il corso di Ebraico e lingue semitiche comparate tenuto nell'anno accademico 1951-1952 nell'allora Istituto di Studi Orientali: «Quegli ambienti poco accoglienti e poco frequentati che mi si presentarono al mio secondo anno di università avevano allora sedici anni, ma nascondevano bene la loro età, perché sembravano già vecchi» ${ }^{1}$, ricorderà lo stesso Garbini, ma a siffatta apparente vetustà si opponeva la giovane promettente età del maestro e dell'allievo: ventinove anni il primo, venti il secondo. Moscati, con le sue notevolissime capacità didattiche, lo introdusse alla storia delle antiche civiltà semitiche nonché allo studio dell'ebraico biblico e della comparazione linguistica semitica; l'anno accademico che seguì, permise anche lo studio della lingua siriaca.

Gli studi orientalistici di Giovanni Garbini videro il loro completamento con il corso di assiriologia di Giuseppe Furlani e quello di arabo di Maria Nallino. La scelta di discutere la tesi di laurea con Sabatino Moscati era ormai sicura e la discussione di laurea ebbe luogo nell'ottobre del 1954 con la correlazione di Giorgio Levi Della Vida. L'argomento della tesi consisteva nella raccolta delle iscrizioni aramaiche antiche con analisi linguistica e grammaticale; la prima stesura fu condotta con l'assidua frequentazione della biblioteca del Pontificio Istituto Biblico e «naturalmente bocciata dal gentile ma severo professore», ma quest' «utile lezione» fece sì che gli altri capitoli della tesi non richiedessero ulteriori seconde stesure ${ }^{2}$.

La conclusione degli studi universitari non ammetteva ormai più deroghe all'adempimento degli obblighi militari e Garbini partì per Lecce il 19 gennaio 1955. Tuttavia, la dinamicità di Moscati - senza soste e che soste non concedeva agli allievi prevedeva che la tesi di Garbini divenisse al più presto uno studio da offrire alla comunità degli studiosi e la consuetudine tra maestro e allievo non si arrestò: nel 1956 fu pubblicata come memoria dell’Accademia Nazionale dei Lincei la monografia L'aramaico antico. Nel medesimo anno il Nostro assunse il ruolo di assistente presso il maestro; furono gli anni, peraltro, della collaborazione all'Enciclopedia dell'arte classica sotto la guida di Ranuccio Bianchi Bandinelli, un'esperienza più volte ricordata da Garbini con piacere.

1 G. Garbini, "Lehrjahre”, in Omaggio a Sabatino Moscati. Testimonianze di allievi e amici, Roma 1992, pp. 11-25: 11

2 Garbini, “Lehrjahre”, p. 13. 
Nel 1960, cioè ad appena ventinove anni, Garbini ottenne la cattedra di Filologia semitica all’Istituto Orientale di Napoli, ma il rapporto con la "Sapienza” non s'interruppe: Garbini continuerà a tenervi corsi e a partecipare alle campagne di scavo organizzate da Sabatino Moscati.

Nel 1977, dietro esortazione di Giovanni Pugliese Carratelli - altro maestro riconosciuto del Nostro - Garbini si trasferì alla Scuola Normale Superiore di Pisa; anche in questo caso, non rescisse d'improvviso i rapporti con l'Ateneo d'immediata provenienza, ma vi mantenne per alcuni anni una supplenza. Alla Scuola Normale fu titolare dell'insegnamento di Epigrafia semitica: un'etichetta eccessivamente limitativa, se vogliamo, e per di più collocata in un contesto didattico privo di materie affini. A queste limitazioni supplirono l'energia e gli interessi molteplici del docente. A lezione, Garbini leggeva e commentava epigrafi, ma trattava anche della comparazione linguistica semitica; inoltre, fu durante questo periodo ch'egli sviluppò in maniera assai approfondita l'interesse per un campo che sino allora aveva affrontato in maniera episodica: gli studi biblici.

Nel novembre 1982, Garbini si spostò nell'università ove aveva compiuto gli studi di laurea: lo volle come suo successore Sabatino Moscati, il quale si trasferiva alla neocostituita Seconda Università di Roma “Tor Vergata”. L’anno seguente, il 30 luglio, Garbini venne anche accolto come socio corrispondente dell'Accademia Nazionale dei Lincei; ne divenne socio nazionale il 6 dicembre 1990.

«Un lavoro scientifico ha come scopo primario e irrinunciabile la novità dei risultati raggiunti; esso deve dire o dare qualcosa che prima non c'era»: a questa regola, enunciata da Moscati, Garbini si è sempre attenuto ${ }^{3}$. Glielo hanno consentito l'applicazione costante dell'acribia e - soprattutto - della sua intelligenza.

Gli studi d'esordio riguardarono la linguistica semitica. Si è già accennato all’iniziale lavoro, frutto della tesi di laurea, sull'aramaico antico: a questa monografia fece seguito Il semitico di nord-ovest (Napoli 1960), i cui presupposti s'innestavano sull'ipotesi moscatiana di una originaria unitarietà delle lingue semitiche di area nordoccidentale. Ben presto, Garbini riconsiderò completamente questa visione nell'ambito di una più vasta ricerca nella altre lingue semitiche (cfr. Le lingue semitiche. Studi di storia linguistica [Napoli 1972; 1984²] e Introduzione alle lingue semitiche [in collaborazione con $\mathrm{O}$. Durand; Brescia 1994]). Le isoglosse che l'arabo manifestava con l'ugaritico anziché con l'accadico collocavano il primo in un contesto più vicino al semitico nordoccidentale. Questo dato indusse Garbini a considerazioni di linguistica storica le quali portarono a una ricostruzione che, a fronte di una fase arcaica rappresentata dall'accadico, l'eblaita e l'ugaritico, riconoscerà il processo innovativo introdotto dalla lingua amorrea; un processo durato circa due millenni e che influenzò le lingue della Siria settentrionale e meridionale (cananaico), fino all'aramaico e all'arabo.

Gli studi linguistici furono in particolare affiancati dall'esame delle testimonianze epigrafiche aramaiche, fenicio-puniche, ebraiche, sudarabiche; mai vi verrà disatteso il proposito di intervenire adducendo nuove esegesi. Questa ricerca condusse a due importanti

3 Garbini, “Lehrjahre”, p. 14. 
sintesi in monografie dedicate all'argomento: nel 1979, con Storia e problemi dell'epigrafia semitica (Napoli) e nel 2006 con l'Introduzione all'epigrafia semitica (Brescia).

Altro campo di ricerca prediletto da Garbini fu quello degli studi biblici. Coerente con il suo temperamento di studioso estremamente attento al vaglio delle fonti, Garbini si trovò a dover discutere i punti più critici delle ricerche bibliche: la questione wellhauseniana delle fonti, la veridicità storica di quanto trasmesso dall'Antico Testamento, la necessità di dover abbassare le datazioni di composizione dei testi, sino alla questione della costituzione di un testo critico della Bibbia ebraica. Le sue posizioni hanno contribuito a far collocare Garbini fra i cosiddetti "minimalisti” dai quali, peraltro, egli teneva a distinguersi «per la loro mancanza di senso filologico e storico che ha impedito loro di scorgere quanta storia non sacra sia nascosta sotto le intenzionali corruttele del testo biblico» ${ }^{4}$. Questa affermazione consente di mette in rilievo due dei più importanti aspetti metodologici di Garbini in merito al testo biblico. L'uno è costituito dalla messa a confronto dei testi biblici con le fonti extrabibliche, un metodo che ha permesso di meglio collocare Israele nel contesto della storia del Vicino Oriente antico. L'altro punto riguarda l'esame "ideologico" dei testi, ossia lo stabilire quanto essi abbiano subito rimaneggiamenti, nel corso della loro trasmissione, dovuti a preoccupazioni di vario ordine, in specie religioso e politico. In questo senso, tra la vastissima produzione di Garbini, hanno valore esemplare Storia e ideologia nell'Israele antico (Brescia 1986) e l'edizione del Cantico dei cantici (Brescia 2010): in quest'ultima, in particolare, è sorprendente l'individuazione di paralleli tra il testo biblico e la poesia greca alessandrina, tanto da definire magistralmente il contesto culturale della composizione.

Lo studio dei testi permise a Garbini di prendere posizione in merito a svariati temi storico-religiosi. Sono molteplici a questo riguardo i contesti culturali toccati da Garbini; per quello fenicio-punico, si pensi al solo titolo del suo libro I Fenici. Storia e religione (Napoli 1980), ma numerosissime attenzioni vennero dedicate anche alle altre culture semitiche. Gli studi biblici hanno goduto di una particolare attenzione sino agli anni più recenti, addirittura con sconfinamenti nell'ambito neotestamentario.

Dieci anni fa il nostro studioso volle ripercorrere i propri interessi di studi con una rassegna ragionata dei suoi scritti, L'opera di Giovanni Garbini. Bibliografia degli scritti 1956-2006 (Brescia 2007): scorrere i suoi titoli - esercizio tutt’altro che tedioso - è il modo migliore per avere una panoramica dei campi trattati. Solo tra le monografie, da allora, se ne sono aggiunte sei (Scrivere la storia d'Israele, Brescia, Paideia 2008; Letteratura e politica nell'Israele antico, Brescia, Paideia 2010; Dio della terra, dio del cielo, Brescia, Paideia 2011; I Filistei. Gli antagonisti di Israele (nuova edizione), Brescia, Paideia 2012; Il Poema di Baal di Ilumilku, Brescia, Paideia 2014; Vita e mito di Gesù, Brescia, Paideia 2015); Il vangelo aramaico di Matteo e altri saggi (Brescia, Paideia 2017). Si tratta, sino a oggi, di una mole di trenta monografie, quasi cinquecento articoli, oltre a numerosissime voci di enciclopedie e recensioni. La sua eredità è cospicua: è stata e sarà feconda per $\mathrm{i}$ futuri studi semitici.

4 G. Garbini, L'opera di Giovanni Garbini, Bibliografia degli scritti 1956-2006, p. 16. 